\title{
A Spectral-Scanning Magnetic Resonance Imaging (MRI) Integrated System
}

\author{
Arjang Hassibi ${ }^{1,2}$, Aydin Babakhani ${ }^{1}$, and Ali Hajimiri ${ }^{1}$ \\ ${ }^{1}$ California Institute of Technology, Pasadena, CA 91125, USA \\ ${ }^{2}$ University of Texas at Austin, Austin, TX 78712, USA
}

\begin{abstract}
An integrated spectral-scanning magnetic resonance imaging (MRI) technique is implemented in a $0.12 \mu \mathrm{m} \mathrm{SiGe} \mathrm{BiCMOS}$ process. This system is designed for small-scale MRI applications with non-uniform and low magnetic fields. The system is capable of generating customized magnetic resonance (MR) excitation signals, and also recovering the MR response using a coherent direct conversion receiver. The operation frequency is tunable from DC to $37 \mathrm{MHz}$ for wide-band MRI and up to $250 \mathrm{MHz}$ for narrow-band MR spectroscopy.
\end{abstract}

\section{INTRODUCTION}

Scaling down magnetic resonance imaging (MRI) [1] and nuclear magnetic resonance (NMR) spectroscopy systems [2] is an exceptionally challenging task. Nonetheless, it realization has always been a goal in medical tomography imaging (3D imaging) due to its huge potential applications in non-invasive imaging for the field of pointof-care $(\mathrm{PoC})$ diagnostics.

To design a truly desktop and eventually hand-held magnetic resonance (MR) system, we are required to scale down all the components of the spectroscopy system, while keeping the sensitivity, detection time, spectral resolution, and the spatial resolution (relative to the sample size) equivalent to the specification of large MR systems.

The reciprocity theorem of electromagnetic theory, states that the electromotive force (emf) voltage induced in the coils of the MR system, $E(t)$, is describe by

$$
E(t)=-\int_{V} \frac{\partial}{\partial t}\left[\vec{B}_{n}(\vec{r}) \cdot \vec{M}(\vec{r}, t)\right] d V
$$

where $\vec{B}_{n}(\vec{r})$ is the magnetic field generated by unit current in the coils at point $\vec{r}$, and $\vec{M}(\vec{r}, t)$ is the magnetic moment (and dipole) density created by the magnetization field $\vec{B}_{0}$ in the sample volume $V$ (see Fig. 1 ).

In scaling down by factor $\alpha, \vec{M}(\vec{r}, t)$ and $E(t)$ are reduced by $1 / \alpha^{3}$, since the number of signal-generating magnetic dipoles (e.g., ${ }^{1} \mathrm{H}$ in proton MR spectroscopy and imaging) is inversely proportional to volume. However, Ampere's Law, indicates that $\vec{B}_{n}(\vec{r})$ is proportional to $\alpha$ to the first order, indicating that smaller coils are more "sensitive". Therefore, by utilizing small-scale coils intimately close to the object, we still experience significant

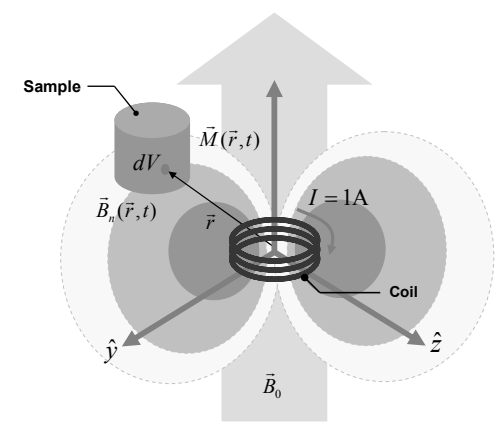

Fig 1: Magnetic resonance spectroscopy system.

signal reduction, with a theoretical lower boundary of $1 / \alpha^{2}$. This concept has been studied extensively in small volume NMR [3] and high-resolution MRI systems [4,5].

On the other hand, $\alpha$ has little effect on $\vec{B}_{0}$ since magnetization can be provided by sample- and coilindependent sources. For spectroscopy applications, it is common to find huge magnetization setups, typically of the super-conducting type, which are able to create up to 10$20 \mathrm{~T}$ to analyze the MR spectrum of small objects. Such approaches are clearly impractical and costly for any PoC application. For portable MR system, it is more appropriate to use small-scale permanent rare-earth magnets which can produce up to $1.5 \mathrm{~T}$ peak magnetic field. Their successful implementation has been previously reported for NMR spectroscopy and surface MR tomography applications $[6,7]$.

Although permanent magnets can be assembled to have a small dimension suitable for PoC systems, the magnetic field surrounding them is very inhomogeneous and nonuniform. Hence, if we place an object containing magnetic dipoles (e.g., ${ }^{1} \mathrm{H}$ with gyromagnetic ratio of $\gamma=42.58$ $\mathrm{MHz} / \mathrm{T}$ ) in their vicinity, the dipoles within the object will demonstrate a wide range of MR frequencies proportional to their local field strength. Based on Torrey-Bloch formulations magnetic gradients significantly reduce the lifetime of the dipoles if, dipoles are subject to diffusion spreading [8]. This means that non-uniform fields generated by permanent magnets not only widen the bandwidth of MR spectrum, but also increases the decay of their MR response. Both of these impediments along with $1 / \alpha^{2}$ to $1 / \alpha^{3}$ degradation in the signal amplitude are fundamental barriers in scaling MR-based systems today. 
In this paper, we show that by leveraging the advantages of integrated circuits, we can address some of the aforementioned challenges. This is done by designing a wide-band MRI system which uses the spectral-scanning MRI technique which exploits the non-uniformity of the magnetic field to acquire tomographic information [9]. We have designed, fabricated, and tested this system in a $0.12 \mu \mathrm{m}$ SiGe BiCMOS process.

\section{IMAGING METHOD SPECIFICATIONS}

The method of detection, spectral-scanning MRI implemented in our system, is based on exploiting the nonuniformity of the magnetic field to create coordinatedependant MR frequencies within the sample [9]. For a given permanent magnet, the magnetic field is non-uniform, but still deterministic. Accordingly, we have a mapping between the coordinates of magnetic dipoles and their MR frequency, as shown in Fig. 2. This method, in principle, is a wideband spectroscopy technique which performs parallel MR analysis to get tomographic information about the magnetic dipole density within the imaging volume. This is also much in contrast with conventional MRI which is essentially a narrowband spectroscopy technique [1].

As mentioned before, to generate the magnetization field $B_{0}$ in small-scale MR systems, rare-earth permanent magnets are used. In such arrangements, the strength of the field is typically less than 1T. The field gradient $G=\nabla B_{0}$, can also vary from 0.01 to $40 \mathrm{~T} \cdot \mathrm{m}^{-1}$ in spatial coordinates where magnetic field strength has $10 \%$ to $\% 80$ of its maximum value at the magnet surface. To give a practical example, such a magnetization setup creates proton $\left({ }^{1} \mathrm{H}\right)$ MR frequency range of $4.2 \mathrm{MHz}$ to $34 \mathrm{MHz}$ and maximum transversal decay of $0.1 \mathrm{msec}$ within the imaging volume based on Torrey-Bloch formulations [8]. Therefore, we require a MR detection system capable of operating within approximately one frequency decade (almost 4-40MHz) and with a spot bandwidth in excess of $10 \mathrm{kHz}$.

One important concern regarding operating at the 4$40 \mathrm{MHz}$ frequency range is the wide-band nature of the detection, which is greater than an octave and closer to a decade. This potentially creates practical challenges in both the signal generation (transmitter) and signal detection circuitry (receiver) of the spectral scanning system due to in-band high-order harmonics. Consequently, we have decided to keep the operation bandwidth of the MR system (both transmitter and receiver) within one octave in our design, however make the system architecture tunable and capable of sweeping its operational frequency to cover the whole 4-40MHz decade.

The basic idea is to simultaneously excite multiple frequencies within the range of interest and detect them with a narrowband system. This parallel analysis is very conducive to the parallel implementation of the system, and significantly increases the imaging and processing speed.

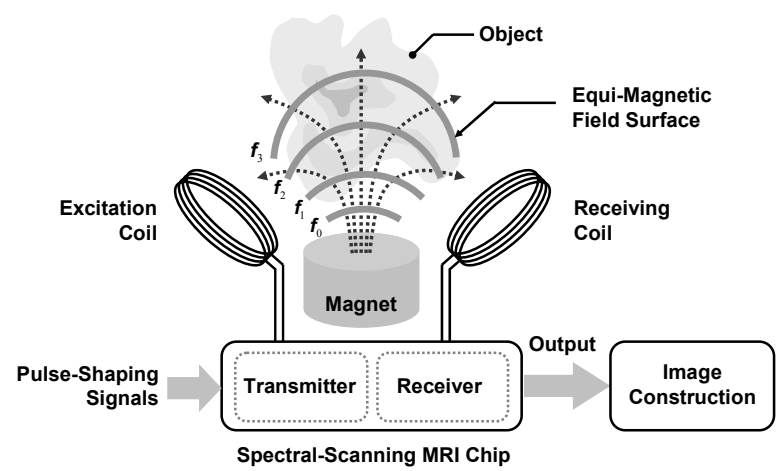

Fig 2: The block diagram of the spectral scanning MRI system, where equi-magnetic field surfaces translate to specific MR resonance frequencies.

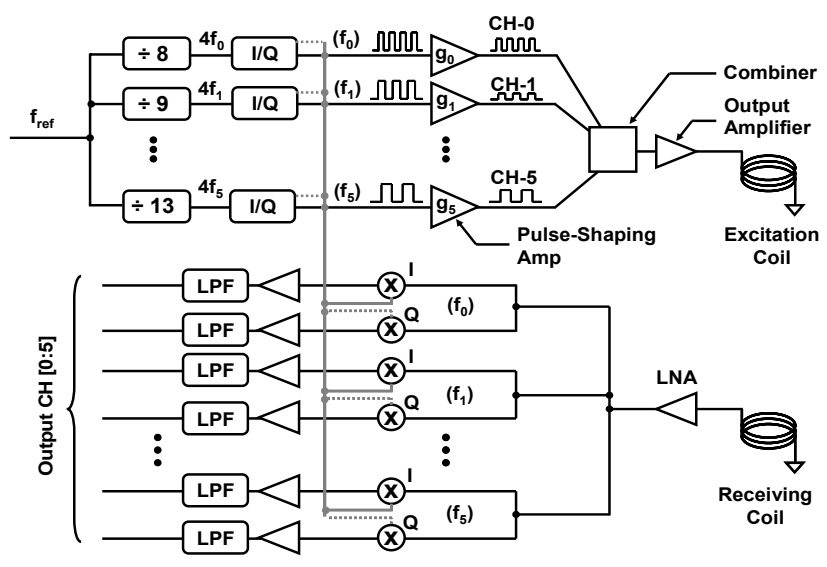

Fig.3: Architecture of the spectral scanning MRI chip.

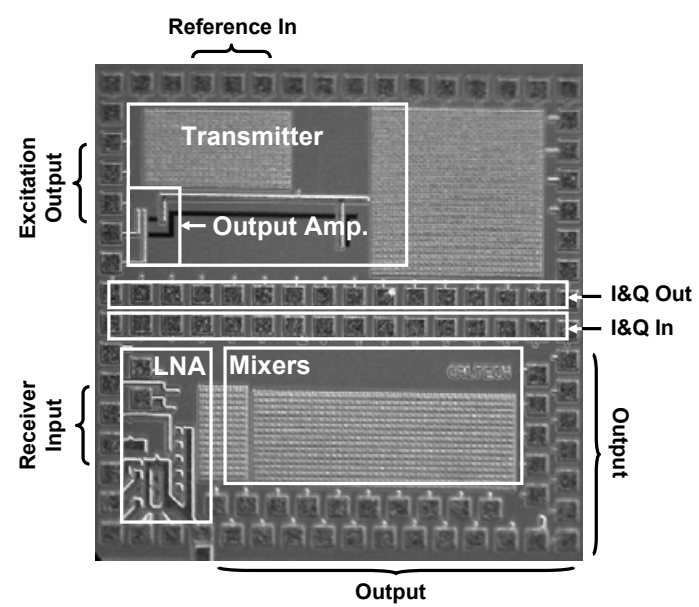

Fig. 4: Chip micrograph

Different equi-magnetic field shells across the sample are mapped to different MR frequencies. To resolve the spatial ambiguity within a shell, (see Fig. 2) multiple measurements are performed while changing the relative position of the object with respect to the magnet. The spectral scanning approach expedites this process substantially. 


\section{CiRcuit ImPlementATION AND RESUltS}

We have illustrated the spectral-scanning MRI system architecture and its die micrograph in Fig. 3 and Fig. 4 respectively. The system consists of the transmitter which generates the customized MR excitation signal (pulses), and the receiver which recovers and coherently detects the MR responses, both integrated on the same die.

Based on the specifications of our system, we are required to generate an excitation spectrum with bandwidth of just short of one octave, yet tunable and capable of covering the whole $4-40 \mathrm{MHz}$. In our design, we have implemented six tunable channels in the transmitter which collectively and when combined create the desired spectrum. In the transmitter (see Fig. 3) the excitation tone signals for all channels are generated by using a reference frequency and digital counters working as frequency dividers. The divisors for the digital counters ( 8 through 13 in our design) are selected such that the output frequencies of all 6 channels stay within one frequency octave and avoid complications related to the second-order harmonics. After the tone generation using the digital dividers, we create the I and Q signals which are used in the transmitter and in the direct conversion receiver. The $\mathrm{I}$ and $\mathrm{Q}$ generator essentially is a divide by 4 counter circuitry, which results in an overall frequency division of $\{32,36,40,44,48,52\}$ in the channels. In Fig. 5 we have illustrated the frequency allocation of each channel within the output spectrum.

Generally in MRI and NMR systems, the excitation sinusoidal pulses have a short duration with time-varying amplitude specifically designed based on the imaging technique and image reconstruction algorithm [1]. Accordingly, to satisfy this requirement in the signal path of each channel we implement a synchronized enabling signal which activates the channel output with maximum switching frequency in excess of $50 \mathrm{MHz}$. The output of this can further be tuned to compensate for amplitude offsets using a PMOS variable resistor as shown in Fig. 6. In Fig. 7, we have shown the output spectrum of Channel-0 (devisor equal to 32) of the transmitter when the $31.25 \mathrm{MHz}$ generated tone is switched on and off using the channel control signal with duration of $20 \mu \mathrm{s}$. The reference frequency in this case is $1 \mathrm{GHz}$.

Next, in order to combine the outputs of all channels and create the excitation spectrum, we use a resistornetwork passive combiner followed by an output amplifier which drives the excitation coils. The maximum peak-topeak voltage of the excitation signal for the combined channels at the combiner output with $\mathrm{V}_{\mathrm{CC}}=1.3 \mathrm{~V}$ is $3 \mathrm{mV}$, which is sufficient for driving the RF amplifiers of the MR systems. The output of the amplifier when all six channels are operating without amplitude modulation (and switching) is shown in Fig. 8 with again the reference frequency of $1 \mathrm{GHz}$. The transmitter operation frequency range is from $\mathrm{DC}$ to $37 \mathrm{MHz}$ corresponding to an input reference frequency of $\mathrm{DC}$ to $1.2 \mathrm{GHz}$, respectively. The measured offset of the channels was approximately $2.3 \mathrm{~dB}$ which can

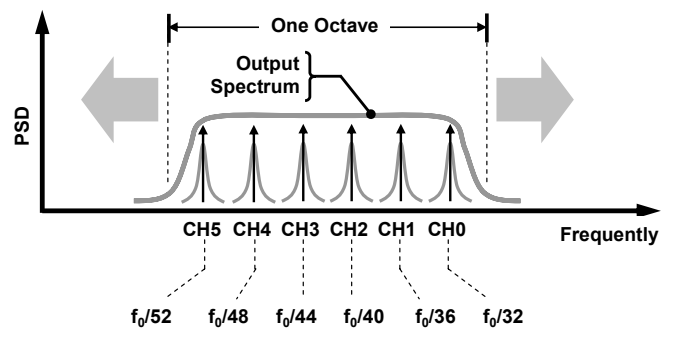

Fig.5: Spectrum profile of the output of the transmitter

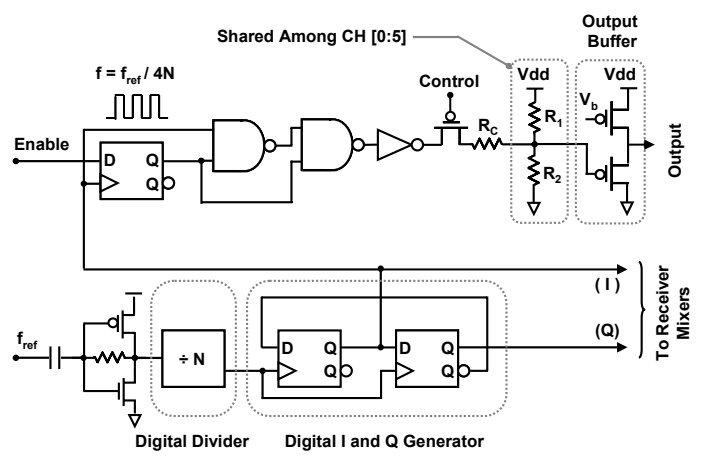

Fig.6: Signal generation circuitry for a single channel of the transmitter.

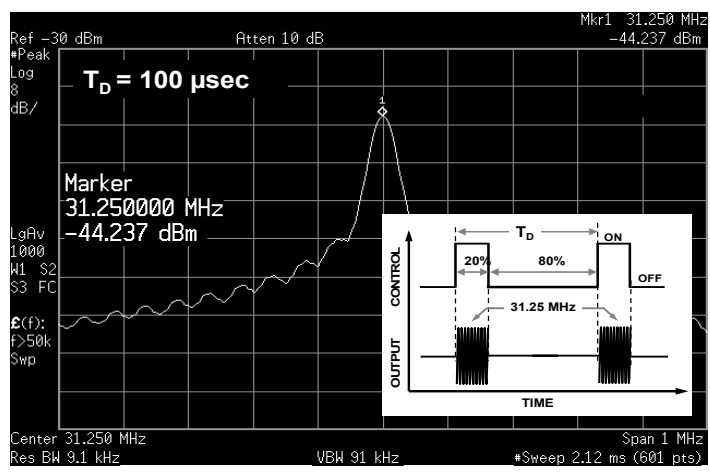

Fig. 8: Typical output spectrum of the transmitter with reference frequency of $1 \mathrm{GHz}$.

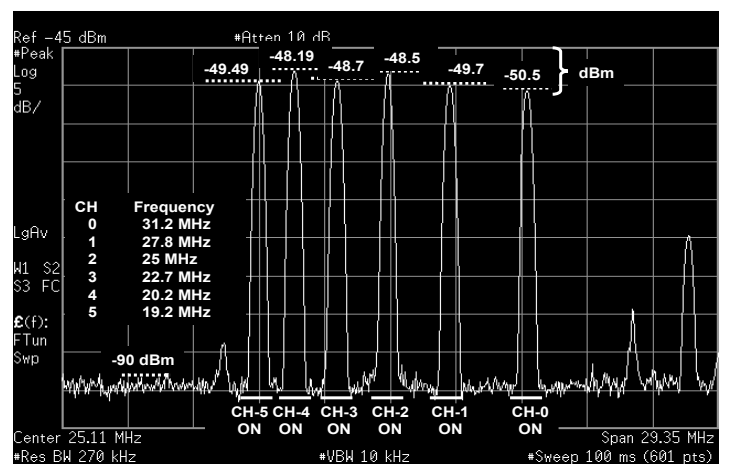

Fig.7: Output spectrum and control signal of Channel-0 in the transmitter.

be calibrated using the PMOS tuning resistor. The overall power consumption with $\mathrm{V}_{\mathrm{CC}}=1.3 \mathrm{~V}$ varies between $6.5 \mathrm{~mW}$ at $\mathrm{DC}$ to $18.2 \mathrm{~mW}$ at $1.2 \mathrm{GHz}$. The transmitter measured performances are listed in Table I. 


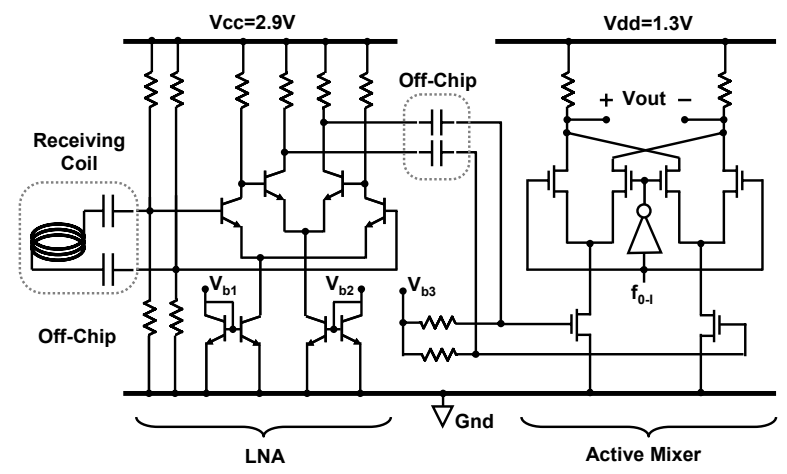

Fig.9: The receiver circuitry for a single channel.

In the receiver, the signal induced into the receiving coils $E(t)$, which is the magnetic dipoles response to the excitation spectrum and $B_{0}$, is initially amplified using a wideband LNA (see Fig. 9). To realize the LNA in our system, we have taken advantage of the bipolar NPN transistors of the SiGe BiCMOS process. The justification for this is the low noise characteristics, low $1 / f$ noise corner, and the high gain of the NPN SiGe transistors. The LNA has a differential two-stage topology with resistive loads. The differential input of the LNA is biased internally, and there are two DC blocking external capacitors which connect the receiving coil to the input of the LNA. It is imperative to understand that the input impedance of the LNA in our design is not matched to the impedance of the coil (mostly inductive with less than $1 \Omega$ resistance) since the LNA is operating as a high input impedance amplifier not a matched amplifier typically used in RF transceivers. The LNA gain and the input referred input noise voltage are $27-31 \mathrm{~dB}$ and $150 \mathrm{pV} / \sqrt{\mathrm{Hz}}$ respectively at the frequency range of $10 \mathrm{MHz}$ to $250 \mathrm{MHz}$. The LNA has an input $1 \mathrm{~dB}$ compression point of $-21 \mathrm{dBV}$ with overall current consumption of $19.2 \mathrm{~mA}$ with $\mathrm{V}_{\mathrm{CC}}=2.9 \mathrm{~V}$.

The induced signal into the coils after amplification by the LNA which amplifies the whole one octave band is introduced to the channel mixers for down conversion as shown in Fig. 3. The mixers in each channel have a CMOS active double-balanced topology with resistive loads. The required LO frequency for the mixers comes from the transmitter block. In each receiving path of all six channels there are two mixers which are driven by I and Q of that corresponding channel. Using this method we are able to achieve $23 \mathrm{~dB}$ differential voltage gain for the mixers, with an input $1 \mathrm{~dB}$ compression point of $-15 \mathrm{dBV}$. The bandwidth of the mixer which basically sets the bandwidth of the receiver chain is approximately $250 \mathrm{kHz}$ with the external capacitive loads.

The overall gain of the receiver is $50 \mathrm{~dB}$ with $1 \mathrm{~dB}$ input compression point of $-42 \mathrm{dBV}$. The total power consumed in the receiver is between $78.2 \mathrm{~mW}$ and $90 \mathrm{~mW}$ depending on the operation frequency from DC to $1.2 \mathrm{GHz}$. In Table I, we have listed all the measured specifications of the system including the transmitter and receiver blocks
In our system, for narrow-band MR spectroscopy we have also included two auxiliary channels which can function independently from the MRI transceiver. The major difference between these two channels and the wideband MRI transceiver is only in their transmitter. They do not have the initial digital dividers and their output is not combined with any other channel. Using these channels narrow-band signal excitation and coherent detection can be carried out up to $250 \mathrm{MHz}$ using the same LNA and mixers. The operation frequency in this mode is limited by the LNA bandwidth of the LNA and matching considerations.

\section{CONCLUSION}

An integrated circuit implementation of a SpectralSacnning MRI is shown for the first time. A coherent multiple frequency transmitter and receiver design provides high sensitivity (equivalent to a lock-in operation). We believe that this SSMRI implementation is a major step toward successful demonstration of a hand-held MRI unit.

TABLE I

SPeCtral SCANNING MRI System SPecifications

\begin{tabular}{|r|l|}
\hline SoC Specifications & \\
\hline Process & IBM $8 \mathrm{HP} 0.12 \mu \mathrm{m}$ SiGe BiCMOS \\
\hline Die specifications & $3 \mathrm{~mm} \times 3 \mathrm{~mm}, 88$ pins $(56 \mathrm{I} / \mathrm{O}, 32$ test $)$ \\
\hline Off-chip & MRI Coils, DC blocking capacitors \\
\hline Power consumption & $78.2-90 \mathrm{~mW}$ \\
\hline${ }^{1} \mathrm{H}$ detection & DC-37MHz, $B_{0}=0-0.85 \mathrm{~T}$ \\
\hline Transmitter & \\
\hline Operation frequency & MRI [DC-37MHz], NMR [DC-250MHz] \\
\hline Number of channels & MRI $[0: 5]$, auxilary $[0: 1]$ \\
\hline Output level & $0-3 \mathrm{mV} /$ channel, $I_{\mathrm{MAx}}=2.2 \mathrm{~mA}$ \\
\hline Modulation frequency & $50 \mathrm{MHz}$ \\
\hline In-band intermods level & $-40 \mathrm{~dB}$ of channel output \\
\hline Power consumption & $6.5-18.2 \mathrm{~mW}$ for DC-1.2 $\mathrm{GHz}$ input frequency \\
\hline Receiver & \\
\hline Operation frequency & $<250 \mathrm{MHz}, \mathrm{BW}=250 \mathrm{kHz}$ \\
\hline LNA & Gain $=27 \mathrm{~dB}$, input noise $=0.15 \mathrm{nV} / \mathrm{Hz} / 2$ \\
\hline Mixer & Gain $=23 \mathrm{~dB}, 1 \mathrm{~dB}$ comp $=-15 \mathrm{dBV}$ \\
\hline Power Consumption & LNA $=55.5 \mathrm{~mW}$, Mixers $=16 \mathrm{~mW}$ \\
\hline &
\end{tabular}

\section{REFERENCES}

[1] E. M. Haacke, R. W. Brown, M. R. Thompson, and R. Venkatesan, Magnetic Resonance Imaging: Physical Principles and Sequence Design, New York, Wiley, 1999.

[2] P. Callaghan, Principles of Nuclear Magnetic Resonance Microscopy, New York, Oxford, 1993.

[3] D.L. Olson et al., "High-Resolution Micro-coil ${ }^{1} \mathrm{H}-\mathrm{NMR}$ for MassLimited, Nanoliter-Volume Samples," Science, vol. 270, pp. $1967-$ 1970, December 1995.

[4] L. Ciobanu et al., "3D Micron-Scale MRI of Biological Cells," Solis Atate Nucl. Magn. Reson., vol 25, pp. 138-141, January 2004.

[5] C. Massin et al., "High-Q factor RF Planar Microcoils for MicroScale NMR Spectroscopy," Sensors \& Actuators A: Physical, vol. 97, pp. 280-288, April 2002.

[6] J. Perlo et al., "High resolution NMR spectroscopy with a portable single-sided sensor," Science, pp. 1279, May 2005.

[7] P. Prado, F. Casanova and B. Blümich, "3D imaging with a singlesided sensor: an open tomograph," Journal of Magnetic Resonance, vol. 166, pp.228-235, February 2004.

[8] V.M. Kenkre, E. Fukushima and D. Sheltraw, "Simple solutions of the Torrey-Bloch equations in the NMR study of molecular diffusion," Journal of Magnetic Resonance, vol. 128, pp. 62-69, September 1997.

[9] Hajimiri et al., U.S. Patent App. No. 11/499,92. 